\title{
Fluorescence quenching of aromatic hydrocarbons by nitroxide radicals: a mechanismatic study
}

\author{
Tarek A. Fayed, ${ }^{\dagger}$ Gunter Grampp, and Stephan Landgraf \\ Institute of Physical and Theoretical Chemistry, Technical University Graz, 8010-Graz, Technikerstr 4/I, Austria
}

\begin{abstract}
The fluorescence quenching of phenanthrene (Phen), 9-cyanophenanthrene (CPhen), 9-cyanoanthracene (CA), perylene (Per), 9,10-dicyanoanthracene (DCA), and 9,10-diphenylanthracene (DPA) using stable nitroxide radicals as quenchers has been studied by steady state and flash photolysis measurements. Both linearity and deviation from linearity in the Stern-Volmer plots have been observed. The active sphere model was used to discuss the upward curvature of the Stern-Volmer plots in case of Per, DCA, and DPA. The bimolecular quenching rate constant $\left(k_{q}\right)$ of Phen, CPhen and CA was found to be diffusion controlled while in other cases it is lower than the diffusion limit. On the basis of flash photolysis measurements as well as the overlap between the emission spectra of hydrocarbons and the absorption spectra of radicals, a resonance energy transfer mechanism is taken place in case of Per, DPA, DCA, and CA. For Phen and CPhen where the energy gap between the first excited singlet and the nearest lower triplet state is small, an induced intersystem crossing was suggested. Finally, the quenching process was discussed in terms of the free energy dependence $(\Delta G)$ of the electron transfer from nitroxide radicals to the excited hydrocarbons.
\end{abstract}

\section{INTRODUCTION}

Quenching of excited singlet and triplet states of aromatic compounds by paramagnetic species is becoming highly important as many photochemical and biological processes have free radicals as intermediates. The quenching property of excited molecules by free radicals are also interesting due to changes of spin multiplicity during quenching process. Due to high stability, nitroxide radicals are convenient for model studies of fluorescence quenching by doublet species. The more recent use of fluorescence quenching by nitroxides is to examine dynamical processes in biochemical systems [1] and as the basis of a method for fluorimetric detection of radicals in condensed phases [2]. Several mechanisms have been proposed for excited state quenching by free radicals, e.g., exchange induced relaxation processes (intersystem crossing and internal conversion) $[3,4]$, electron transfer $[5,6]$ and energy transfer interactions $[7,8]$. The energy transfer can occur efficiently because both the radiative and absorptive transitions $\left({ }^{1} \mathrm{M}^{*} \rightarrow{ }^{1} \mathrm{M}\right)$ and $\left({ }^{2} \mathrm{R} \rightarrow{ }^{2} \mathrm{R}^{*}\right)$ are spin allowed. While the mechanism of triplet quenching is interpreted by triplet-doublet energy transfer and enhanced intersystem crossing, the fluorescence quenching mechanism still requires more information $[9,10]$.

In this study the fluorescence quenching of some aromatic hydrocarbons, such as phenanthrene (Phen), 9cyanophenanthrene (CPhen), 9-cyanoanthracene (CA), perylene (Per), 9,10-dicyanoanthracene (DCA), and 9,10diphenylanthracene (DPA), by 2,2,6,6-tetramethyl1-1piperidinyloxyl (TEMPO) and its 4-hydroxy derivative (HTEMPO) as well as di-t-butylnitroxide (DTBN) radicals (Scheme 1), has been studied by steady state and flash photolysis measurements in different solvents. Our objective is to assess the contribution of electron

†On leave from Chemistry Department, Faculty of Science, Tanta University, Tanta, Egypt. transfer to quenching of the excited singlet state of the concerned hydrocarbons by nitroxide radicals.<smiles>CC1(C)CCCC(C)(C)N1O</smiles>

TEMPO<smiles>CC1(C)CC(O)CC(C)(C)N1[O]</smiles>

HTEMPO<smiles>CC(C)(C)N([O])C(C)(C)C</smiles>

DTBN
Scheme

\section{MATERIALS AND METHODS}

The aromatic hydrocarbons (Aldrich except for DCA, Kodak) were purified by sublimation (Phen) or recrystallization from either ethanol or acetonitrile. The nitroxide radicals were from Aldrich and used without further purification. The solvents used (Fluka) were dried over molecular sieve ( $3 \AA$ ) followed by distillation under nitrogen. The absorption and fluorescence spectra were recorded by using a Shimadzu UV-3101 PC spectrophotometer and ISA Fluoromax-2 spectrometer, respectively. The flash photolysis experiments were carried out by using a home-built conventional flash photolysis equipped with a flash lamp having $2 \mu$ s pulse duration. The fluorescence lifetime was measured using ultrabright light emitting diodes as discribed elsewhere [11]. The sample solutions $\left(5 \times 10^{-6} \mathrm{~mol} \mathrm{dm}{ }^{-3}\right)$ were deoxygenated by purging with nitrogen for about $10 \mathrm{~min}$. The concentration of the quencher (nitroxide radicals) was in the range of 3 to $24 \mathrm{mM}$

\section{RESULTS AND DISCUSSION}

The efficiency of fluorescence quenching was determined from steady state fluorescence measurements. The fluorescence intensities have been correlated to the 
Table 1. Fluorescence lifetime $\tau_{f}(n s)$ and bimolecular quenching constant $K_{q}\left(10^{9} \mathrm{M}^{-1} \mathrm{~s}^{-1}\right)$ in different solvents.

\begin{tabular}{|c|c|c|c|c|c|c|c|c|}
\hline \multirow{3}{*}{ Fluorophore } & \multicolumn{4}{|c|}{$\mathrm{CH}_{3} \mathrm{CN}$} & \multicolumn{2}{|c|}{$\mathrm{CH}_{3} \mathrm{OH}$} & \multicolumn{2}{|c|}{ Cyclohexane } \\
\hline & & & $K_{q}$ & & & $K_{q}$ & & $K_{q}$ \\
\hline & $\tau_{f}$ & TEMPO & DTBN & HTEMPO & $\tau_{f}$ & TEMPO & $\tau_{f}$ & TEMPO \\
\hline Per & 6.8 & 4.1 & 4.3 & 3.1 & 5.8 & 3.5 & 4.2 & 3.6 \\
\hline DPA & 8.0 & 5.5 & 5.8 & 3.5 & 8.1 & 5.8 & 8.3 & 1.8 \\
\hline DCA & 13.2 & 4.8 & 4.8 & 3.1 & 13.3 & 4.8 & 11.2 & 3.0 \\
\hline CA & 16.5 & 23.9 & 23.6 & 19.5 & 13.7 & 20.3 & 12.9 & 13.2 \\
\hline Phen & 60.7 & 10.1 & 9.1 & 7.6 & 54.4 & 5.9 & 57.5 & 7.4 \\
\hline Cphen & 22.0 & 18.4 & 16.7 & 15.3 & 22.0 & 12.1 & 28.7 & 9.7 \\
\hline
\end{tabular}

radical concentration using the Stern-Volmer relation [12]

$$
\frac{I_{0}}{I}=1+K_{\mathrm{SV}}[Q]=1+k_{q} \tau_{f}[Q]
$$

where $I_{0}$ and $I$ are the fluorescence intensities in absence and presence of quencher, respectively. $K_{S V}$ is the Stern-Volmer constant and $[Q]$ is the quencher concentration. $k_{q}$ is the bimolecular quenching rate constant and $\tau_{f}$ is the fluorescence lifetime without quencher.
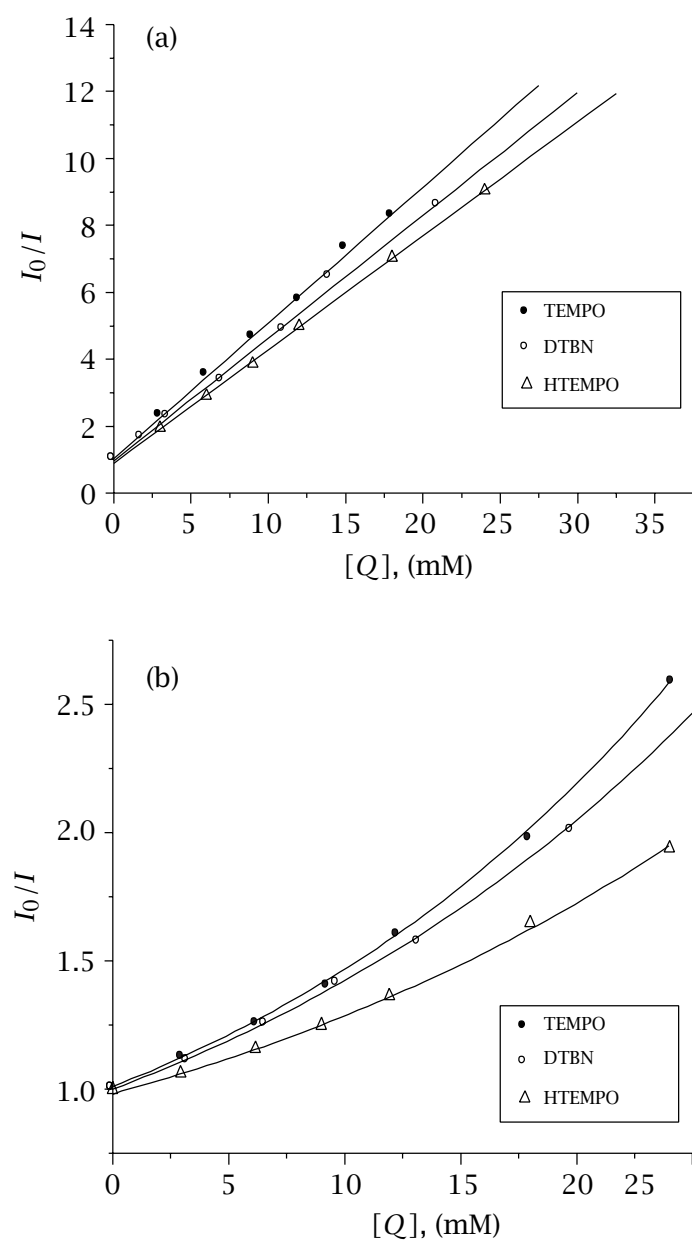

Figure 1. Stern-Volmer plots for fluorescence quenching of (a) CPhen and (b) DPA in $\mathrm{CH}_{3} \mathrm{CN}$ using nitroxide radicals as quenchers.
Representative Stern-Volmer plots are shown in Figure 1. It has been observed that the plots are linear in case of Phen, CPhen, and CA, while they show an upward curvature, at radical concentrations higher than $9 \mathrm{mM}$, in case of Per, DPA, and DCA. In such cases, $K_{\mathrm{SV}}$ values were calculated from the linear part of the plots. The bimolecular rate constants $\left(k_{q}\right)$ calculated from $K_{\mathrm{SV}}$ and the measured $\tau_{f}$ are presented in Table 1.

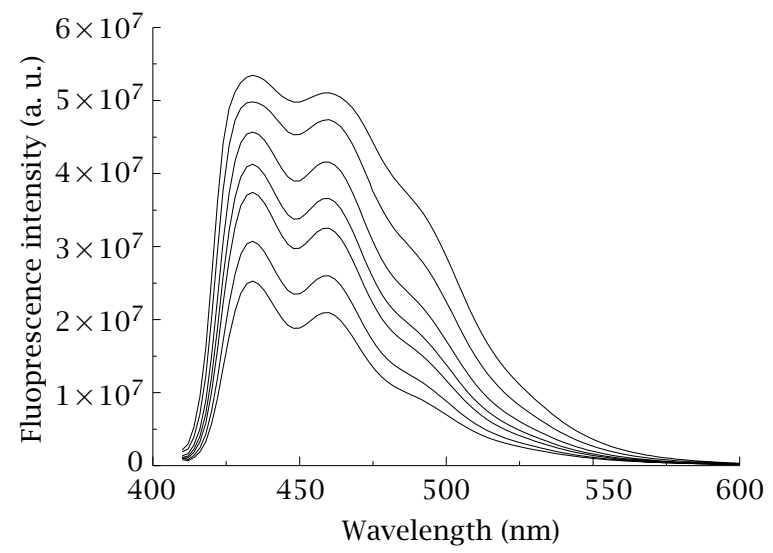

Figure 2. Fluorescence spectra of DCA $\left(5 \times 10^{-6} \mathrm{~mol} \mathrm{dm}^{-3}\right.$, $\lambda_{\mathrm{ex}}=400 \mathrm{~nm}$ ) recorded in $\mathrm{CH}_{3} \mathrm{CN}$ in presence of different concentrations of TEMPO. The concentrations of TEMPO at decreasing fluorescence intensities are; 0.0, 1.5, 3.0, 4.5, 6.0, 9.0 and $12.0 \mathrm{mM}$.

In the absence of any chemical reaction of the excited fluorophore, the positive deviation from the SternVolmer relation may be due to ground state complex formation or transient quenching [13]. Deviation due to ground state complex formation is ruled out since no changes were observed in the absorption spectra of the hydrocarbons after addition of nitroxide radicals. Also, the decrease of the fluorescence intensity (see Figure 2) is not accompanied by appearance of any new band which discards the possibility of exciplex formation. Hence, transient quenching due to the active sphere model [13] can be applied to explain such behaviour. According to this model

$$
\frac{I_{0}}{I}=\exp ^{K_{T}}[Q] \quad \text { or } \quad \ln \frac{I_{0}}{I}=K_{T}[Q]=\frac{V N_{A}}{1000}[Q],
$$


Table 2. Radii of the quenching sphere (in Å) for hydrocarbons in various solvents.

\begin{tabular}{lccccr}
\hline & \multicolumn{3}{c}{$\mathrm{CH}_{3} \mathrm{CN}$} & $\mathrm{CH}_{3} \mathrm{OH}$ & $\begin{array}{r}\text { Cyclohexane } \\
\text { Fluorophore }\end{array}$ \\
\hline TEMPO & DTBN & HTEMPO & TEMPO & TEMPO \\
\hline DPA & 22.6 & 22.6 & 20.3 & 19.9 & 18.1 \\
DCA & 25.1 & 24.4 & 22.4 & 26.5 & 19.0 \\
\hline
\end{tabular}

where $K_{T}$ is the transient quenching constant, $N_{A}$ is Avogadro's number and $V$ is the volume of the active sphere. If a quencher (one or more molecules) exists within this volume at instant that the fluorophore becomes excited, static quenching is assumed to occur instantaneously. According to equation (2) the plot of $\ln I_{0} / I v s$. [Q] is expected to be linear passing through the origin. As an example, Figure 3 shows such plots for the quenching of DPA by nitroxide radicals in $\mathrm{CH}_{3} \mathrm{CN}$ (Per and DCA show similar behaviour). The radii of the active sphere were calculated from the values of $K_{T}$ and shown in Table 2 . The calculated radius of quenching sphere is in the range of 18 to $29 \AA$ which is much greater than the values for the collisional quenching (4$6 \AA$ ) [13].

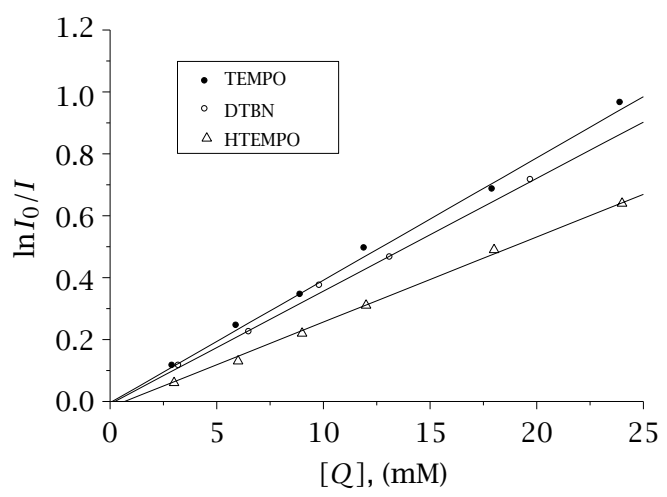

Figure 3. Plots of $\ln I_{0} / I$ vs. radical concentration for the quenching of DPA in $\mathrm{CH}_{3} \mathrm{CN}$.

Since TEMPO, DTBN, and HTEMPO absorb radiation appreciably over the emission maxima of Per, DPA, DCA and CA (see Figure 4) resonance energy transfer can be considered as a possible mechanism for quenching of the excited singlet state of these hydrocarbons. The energies of the first excited singlet states of Per, DPA, DCA, and CA are in the range of 66.7 to $71.2 \mathrm{kcal} \mathrm{mol}^{-1}$, while the doublet energies of the radicals estimated from the first absorption bands are about $47 \mathrm{kcal} \mathrm{mol}^{-1}$. The absorption band of nitroxide radicals around 460 and $477 \mathrm{~nm}$ in $\mathrm{CH}_{3} \mathrm{CN}$ and cyclohexane, respectively, is so broad that the spectral overlap between the fluorescence of the hydrocarbon molecules and the absorption of the radicals is significant $[7,8]$. Since nitroxide radicals are nonfluorescent, the energy transfer mechanism cannot be confirmed by doublet-doublet emission. Flash photolysis measurements with Per, DPA, DCA, and CA in $\mathrm{CH}_{3} \mathrm{CN}$ show a decrease in the absorbance of triplet-triplet absorption immediately after flashing upon addition of TEMPO.
The triplet decay was almost constant. This implies that fluorescence quenching of these hydrocarbons is not due to intersystem crossing.

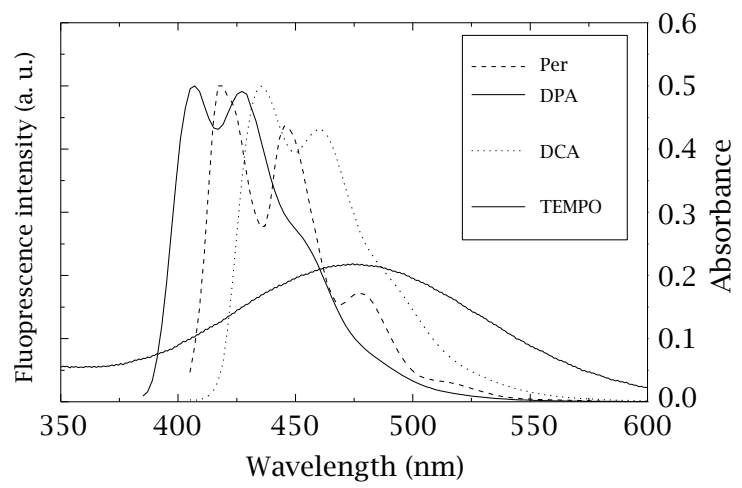

Figure 4. Normalized fluorescence spectra of Per, DPA and DCA as well as the absorption spectrum of TEMPO $(0.02 \mathrm{M})$ measured in $\mathrm{CH}_{3} \mathrm{CN}$.

For Phen and CPhen, where there is no overlap between the emission spectra of both hydrocarbons and the absorption spectra of radicals, and in correspondence with the higher energies of their first excited triplet states an induced intersystem crossing is highly contributing to fluorescence quenching in such cases [14]. The energy of the first excited triplet state is 62.13 and $58.30 \mathrm{Kcal} \mathrm{mol}^{-1}$ for Phen and CPhen, respectively, which is much higher compared to 41.43 and $41.97 \mathrm{kcal} \mathrm{mol}^{-1}$ for DPA and DCA, respectively [15]. The higher energy of triplet states in Phen and CPhen renders the energy gap between the excited singlet state and the nearest lower triplet state smaller, thus enhancing intersystem crossing [15].

The lack of observation of transients other than the triplet makes the elucidation of the quenching mechanism more difficult. However, the role of charge transfer in quenching of excited singlet state of the concerned hydrocarbons can not be neglected. To find out whether such a reaction is thermodynamically feasible in the excited state, the free energy change $(\Delta G)$ has been calculated using the Weller equation [16]

$$
\Delta G=E_{1 / 2}^{\mathrm{ox}}-E_{1 / 2}^{\mathrm{red}}-E_{0,0}^{*}-\frac{e^{2}}{4 \pi \epsilon_{0} \epsilon_{s} R},
$$

where $E_{1 / 2}^{\mathrm{ox}}$ and $E_{1 / 2}^{\mathrm{red}}$ are the oxidation and reduction potentials of the donor (nitroxide radicals) and the acceptor (hydrocarbons) respectively. $E_{0,0}^{*}$ is the energy of the singlet-singlet transition of the excited donor or acceptor. The fourth term is the Coulomb stabilization. 
Table 3. Redox potentials and energy of the lowest $(0,0)$ excited singlet transition as well as the free energy change $(\Delta G)$ for the electron transfer reaction from nitroxide radicals to the excited hydrocarbons in $\mathrm{CH}_{3} \mathrm{CN}$.

\begin{tabular}{lcccccr}
\hline Hydrocarbon & Per & DPA & DCA & CA & Phen & CPhen \\
\hline$E_{1 / 2}^{\text {red }}(V)^{a}$ & -1.67 & -1.94 & -0.98 & -1.58 & -2.44 & -1.88 \\
$E_{0,0}^{*}(\mathrm{eV})$ & 2.82 & 3.16 & 2.89 & 2.97 & 3.57 & 3.62 \\
\hline Quencher & \multicolumn{7}{c}{$\Delta G(\mathrm{eV})$} \\
\hline DTBN $(0.56)$ & -0.59 & -0.66 & -1.36 & -0.83 & -0.57 & -1.18 \\
TEMPO $(0.63)$ & -0.52 & -0.59 & -1.29 & -0.76 & -0.50 & -1.11 \\
HTEMP $(0.68) \mathrm{O}$ & -0.47 & -0.54 & -1.24 & -0.71 & -0.45 & -1.06 \\
\hline
\end{tabular}

Values in parenthesis are the oxidation potentials of radicals and were taken from [6]

${ }^{a}$ Data were taken from Reference [19].

In highly polar solvents such as $\mathrm{CH}_{3} \mathrm{CN}$ this term is negligible [17].

As seen from Table 2, the electron transfer reaction from the radicals to the excited hydrocarbons is highly exothermic and energetically favourable. Also, in all cases the rate constants are found to be the smallest for HTEMPO, which may be due to low $\Delta G$ values. In addition, the bimolecular quenching rate constant decreases with decreasing solvent polarity. In fact, the electron transfer mechanism can also explain the higher values of $k_{q}$ in case of CA and CPhen compared to DCA. According to Marcus theory [18], the rate of electron transfer reaction decreases in the highly exergonic region. Thus, we suggest that electron transfer is playing a significant role in quenching of Per, DPA, DCA, CA, Phen, and CPhen excited singlets.

\section{ACKNOWLEDGEMENTS}

The Technical University Graz and the Deutsche Forschungsgemeinschaft are acknowledged for financial support.

\section{REFERENCES}

[1] J. Matko, K. Ohki, and M. Edidin, Biochemistry 31 (1992), 703.

[2] V. N. Blough and J. D. Simpson, J. Am. Chem. Soc. 110 (1988), 1915.

[3] J. A. Green, L. A. Singer, and J. H. Parks, J. Chem. Phys. 58 (1973), 2690.

[4] A. R. Watkins, Chem. Phys. Lett. 70 (1980), 262.
[5] V. A. Kuzmin, A. S. Tatikolov, and U. E. Borisevich, Chem. Phys. Lett. 53 (1978), 52.

[6] A. Samanta and P. V. Kamat, Chem. Phys. Lett. 199 (1992), 635.

[7] J. S. Puskin, A. I. Vistnes, and M. T. Coene, Arch., Biochem. and Biophys. 206 (1981), 164.

[8] S. Kohtani, M. Murata, and M. Itoh, Chem. Phys. Lett. 247 (1995), 293.

[9] T. Suzuki and K. Obi, Chem. Phys. Lett. 246 (1995), 130.

[10] S. H. Herbelin and N. V. Blough, J. Phys. Chem. B:102 (1998), 8170.

[11] S. Landgraf and G. Grampp, J. Inf. Recording 24 (1998), 141.

[12] J. R. Lakowicz, Principles of Fluorescence Spectroscopy, p. 260, Plenum, New York, ,1986.

[13] N. J. Turro, Modern Molecular Photochemistry, University Science Books, USA, 1991.

[14] J. Karpiuk and Z. R. Grabowski, Chem. Phys. Lett. 160 (1989), 451.

[15] M. Komfort, J. Küster, and H. Dreeskamp, Z. Naturforsch 49a (1994), 578.

[16] D. Rehm and A. Weller, Isr. J. Chem. 8 (1970), 259.

[17] A. Weller, Z. Phys. Chem. NF 133 (1982), 93.

[18] R. A. Marcus and S. Sutin, Biochem. Biophys. Acta 811 (1985), 265.

[19] S. L. Murov, I. Carmichael, and G. L. Hug, Handbook of Photochemistry, 2nd ed., Marcel Dekker, Inc., 1993. 


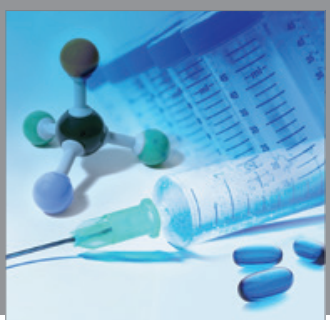

International Journal of

Medicinal Chemistry

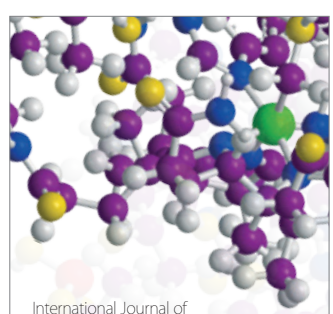

Carbohydrate Chemistry

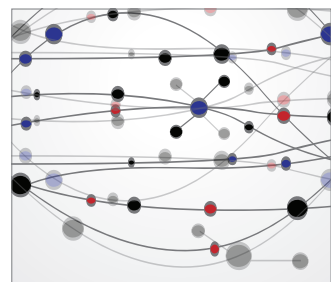

The Scientific World Journal
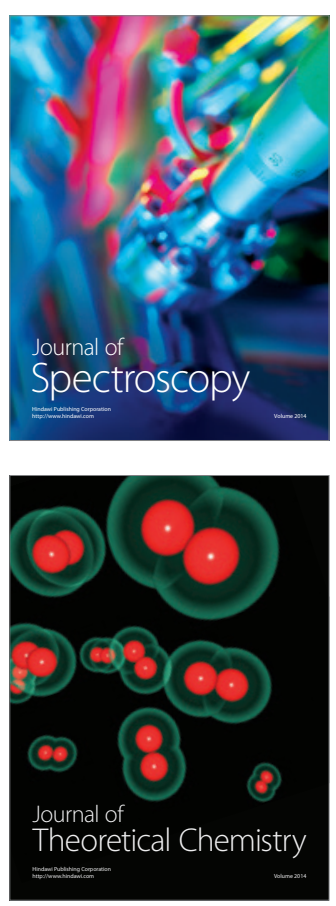
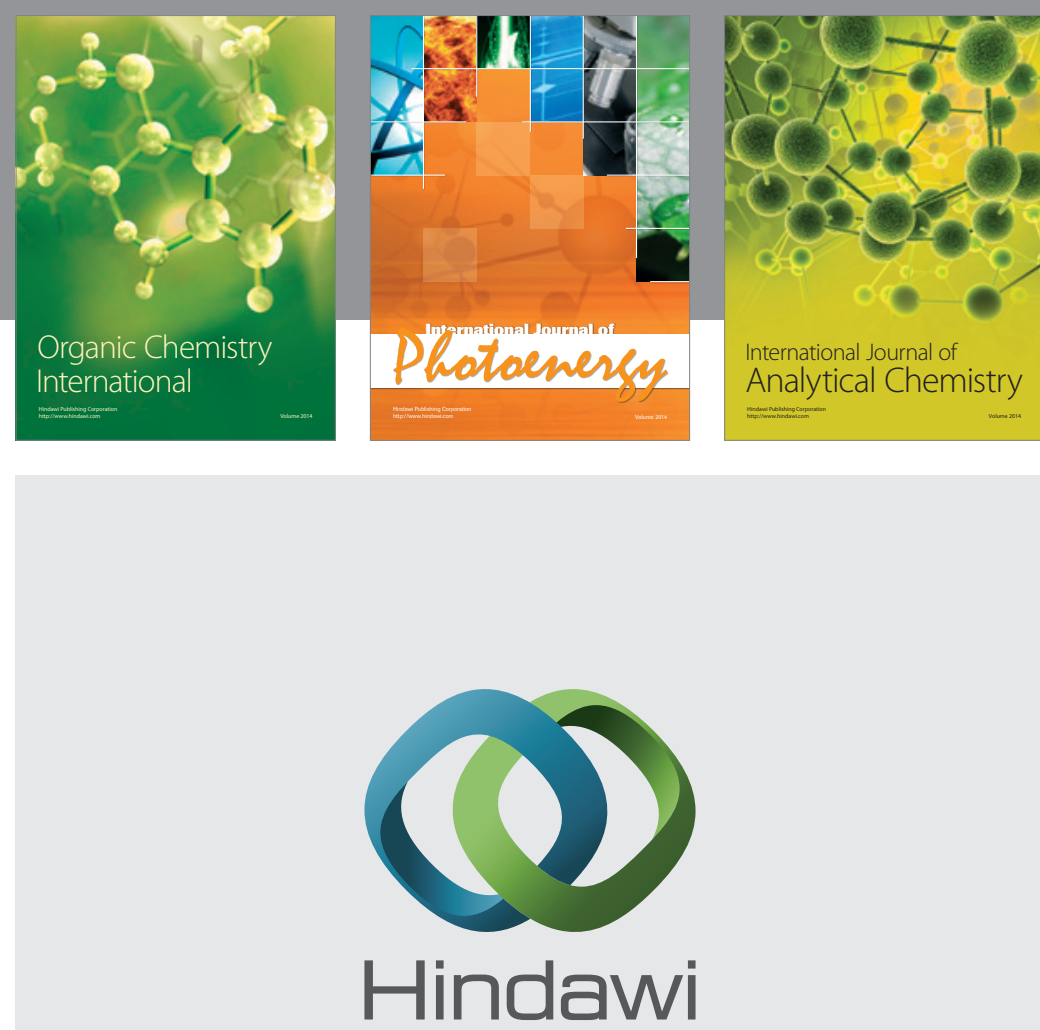

Submit your manuscripts at

http://www.hindawi.com
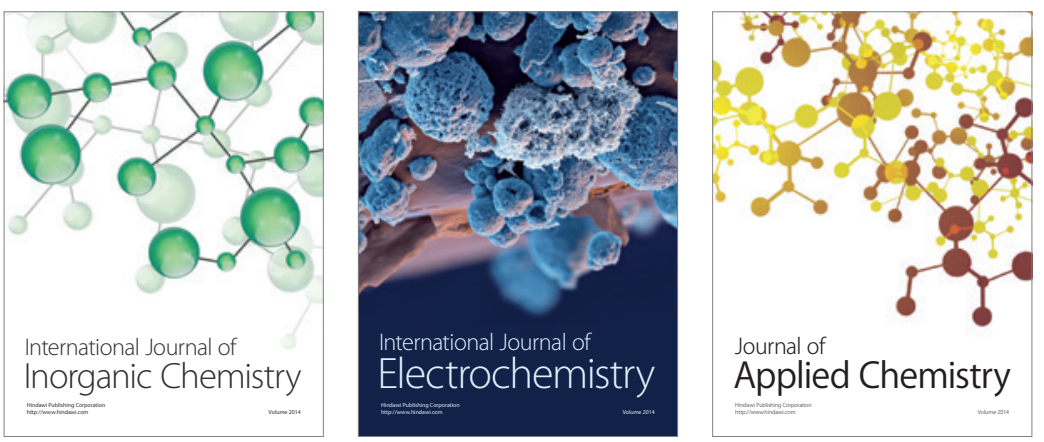

Journal of

Applied Chemistry
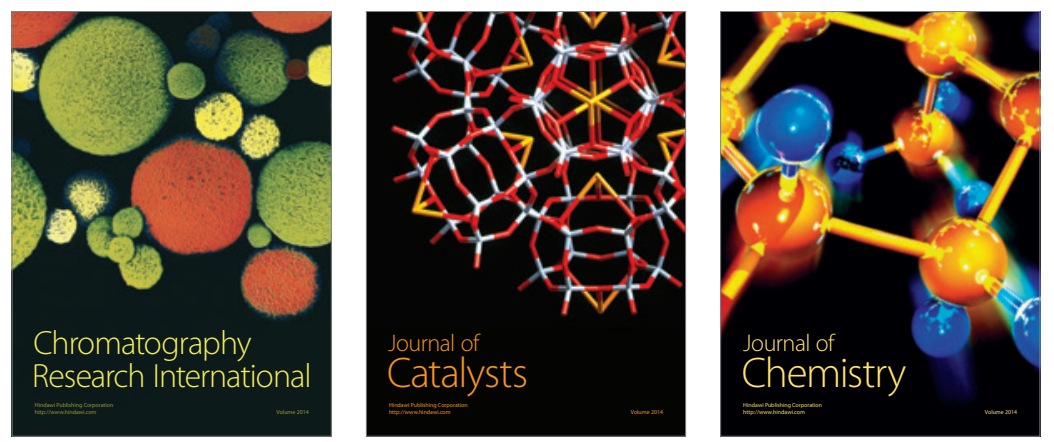
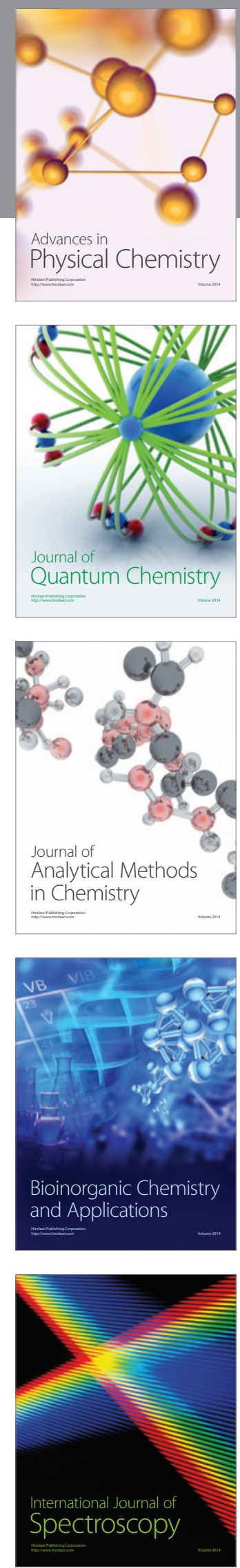